\title{
Autotransplantation for the Management of Teeth with Severe Alveolar Bone Resorption: A Case Report
}

Ji Suk Shim, Jin-hong Park and Joo-Hee Shin *

Department of Conservative Dentistry, Korea University Guro Hospital, Korea

"Corresponding author: Joo-Hee Shin, DDS, MSD, PhD, Clinical associate professor, Department of Conservative Dentistry, Korea University Guro Hospital, \#148, Gurodong-ro, Guro-gu, Seoul 152-703, Korea, Tel: 82-2-2626-1521; Fax: 82-2-837-6245; E-mail: endoshin11@gmail.com

Received date: June 08, 2017; Accepted date: June 26, 2017; Published date: July 03, 2017

Copyright: (C) 2017 Shim SJ, et al. This is an open-access article distributed under the terms of the Creative Commons Attribution License, which permits unrestricted use, distribution, and reproduction in any medium, provided the original author and source are credited.

\begin{abstract}
Background: In comparison with implant treatment, autotransplantation offers potential benefits associated with the use of natural teeth. The periodontal benefits of autotransplantation include bone regeneration and reestablishment of the alveolar ridge, indicating that the procedure can be effective in regions with severe bone loss.

Case Description: A 30-year-old man presented with a chief complaint of tooth pain and mobility. On examination, chronic localized periodontitis and a periapical lesion with vertical bone loss were observed. Following extraction, a third molar was autotransplanted to replace the tooth as an alternative to implant placement, followed by root canal treatment and restoration with a full crown. The patient was followed up for 4 years, during which the probing depth and mobility decreased and the periodontal ligament space and lamina dura were maintained, with no clinical discomfort or inflammation and, importantly, an increased level of alveolar bone on radiographs.
\end{abstract}

Conclusions: Autotransplantation can be an optimal treatment for the replacement of teeth surrounded by severe alveolar bone resorption if the donor tooth and patient conditions are satisfactory.

Practical Implications: Although autotransplantation has relatively strict indications and a lower success rate, the disadvantages can be overcome through prudent case selection and judicious maintenance of periodontal health.

Keywords Autotransplantation; Bone regeneration; Localized periodontitis; Implant

\section{Introduction}

Autotransplantation is defined as the transplantation of embedded, impacted, or erupted teeth from one site into an extraction site or surgically prepared sockets in the same individual [1]. Successful autotransplantation was first reported in the 1950s [2,3] since then, numerous studies on this technique have been conducted and many teeth have been autotransplanted. Although it is a well-established procedure and has proven to be a highly successful rehabilitation technique, [4-7] it is rarely performed in the clinic setting because of advances in osseointegrated implant procedures [8].

Recently, some authors have emphasized the potential benefits associated with the use of natural teeth in autotransplantation instead of artificial materials [9] They demonstrated, for example, that maintenance of the periodontal ligament (PDL) after autotransplantation [10] may render it as an attractive treatment option for young patients in the growth phase, in whom osseointegrated implants are contraindicated because of the possibility of bone and implant fusion without an intervening PDL and inability of the implant to erupt and align with adjacent teeth [11] In addition to maintenance of the PDL, the periodontal benefits of autotransplantation, including bone regeneration and reestablishment of the alveolar ridge, are unique potential benefits of autotransplantation, [9] indicating that the procedure can be effective in regions with severe bone loss. However, few studies have assessed these benefits.

Here we report a case of successful autotransplantation to replace a missing tooth in a region of severe alveolar bone loss in a 30 -year-old man.

\section{Case Report}

A 30-year-old man visited our clinic with a chief complaint of mobility and pain in the mandibular right second molar. On clinical examination, signs of chronic localized periodontitis, including bleeding on probing, a buccal probing depth of $>12 \mathrm{~mm}$ around the middle third, and mobility of $>1 \mathrm{~mm}$ in all directions, were observed. A radiograph revealed vertical alveolar bone resorption and a periapical lesion opening into a buccal fistula, which further compromised the tooth. Endodontic treatment was considered difficult because of the presence of pretreated posts (Figure 1). 


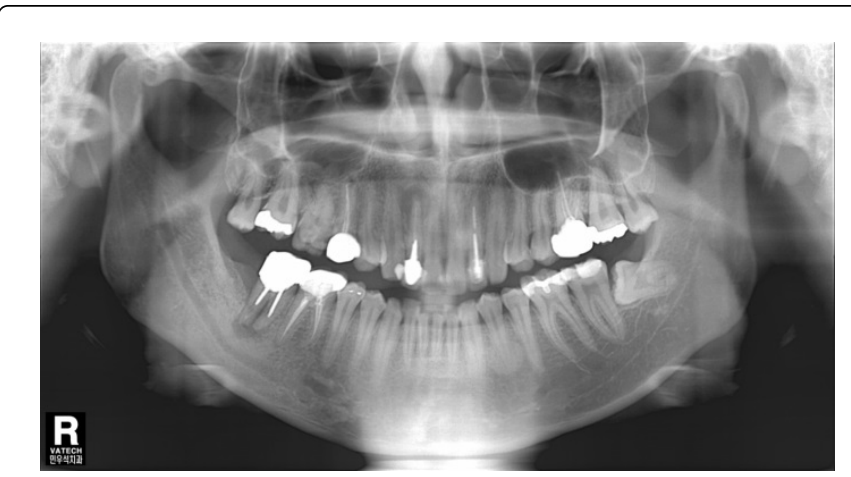

Figure 1: A panoramic radiograph revealing chronic localized periodontitis and a periapical lesion with vertical alveolar bone resorption around the mandibular right second molar.

Extraction followed by autotransplantation was planned. The procedure was performed in a single sitting under local anesthesia. The involved tooth was carefully extracted with forceps, the periapical granulation tissue was curetted, and the recipient site was irrigated with copious amounts of saline. The donor tooth was placed in the recipient site and splinted with stainless steel wire to achieve flexible stabilization. Amoxicillin (500 mg three times a day) and a $0.1 \%$ chlorhexidine rinse were prescribed for a week.

A week after the surgical procedure, root canal treatment was performed to prevent root resorption. The splint was removed 4 weeks after surgery and the root canals were temporarily filled with calcium hydroxide. Following confirmation of a decrease in mobility (Figure 2),

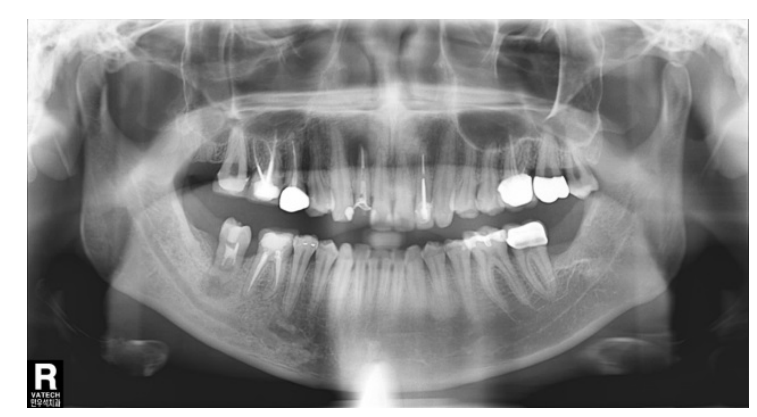

Figure 2: A panoramic radiograph obtained 4 months after autotransplantation. The rigid wire splint has been removed, and the root canals have been temporarily filled with calcium hydroxide. Mobility has significantly decreased 4 months after autotransplantation.

The canals were obturated using the continuous wave technique 4 months after autotransplantation. The donor tooth was restored with a full crown (Figure 3).



Figure 3: A panoramic radiograph obtained 13 months after transplantation. The root canal treatment and crown restoration are completed.

Clinical and radiographic follow-up examinations were conducted every 6 months for a period of 4 years (Figure 4 ).

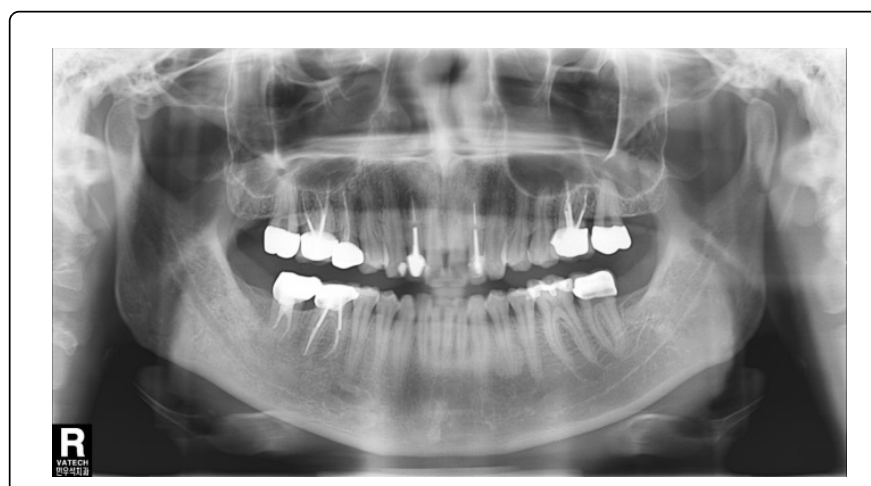

Figure 4: A panoramic radiograph obtained 4 years after transplantation. Note the distal part of the transplanted tooth. The lamina dura is more apparent and the bone height has increased compared with that in Figures 2 and 3.

Throughout the 4-year follow-up period, the patient showed signs of successful autotransplantation, fulfilling all the criteria for successful autotransplantation $[9,12]$ including a decrease in probing depth and mobility and restoration of the periodontal ligament (PDL) space and lamina dura. There was no clinical discomfort or inflammation. Most importantly, a dramatic increase was observed in the level of alveolar bone, particularly on the buccal and distal sides.

\section{Discussion}

This case shows successful autotransplantation to replace a tooth extracted because of severe surrounding bone loss in a 30 -year-old male. Despite continuous developments in implant procedures, placement of implants in patients with severe bone resorption remains a challenge because the predictability of bone grafts decreases as the size of the defect increases, particularly in patients requiring reconstruction of vertical bony defects $[13,14]$. Because our patient had vertical bony defects and was considered a difficult case for implant procedures, autotransplantation was attempted, which gave optimal results including restoration of periodontal health and an increased level of alveolar bone. 
When compared with implant treatment, autotransplantation has several advantages, including a decreased treatment duration, requirement of fewer materials, quicker healing, maintenance of the PDL, and bone regeneration [9]. However, it cannot be a first-line treatment option to replace a tooth in most cases because of disadvantages such as a lower success rate, more strict prerequisites, and technical sensitivity [15]. Case selection is important for successful autotransplantation, and the advantages and disadvantages should be weighed for individual cases.

The common complication of autotransplantation is root ankylosis, and the primary reason for failure is root resorption [16]. However, these factors may not be crucial to overall treatment planning for teeth with severe bone resorption if periodontal health recovers appropriately. This is because implant treatment can be performed at a later stage in recipient areas with a restored periodontium even if the transplant fails because of root resorption. Autotransplantation comes with the potential benefits of bone regeneration and reestablishment of the alveolar ridge [9]. Furthermore, a study demonstrated maintenance of periodontal health after autogenous autotransplantation, [17] and few studies on the success rates of autotransplantation have pointed out periodontal problems as a complication or the reason for failure.

Successful autotransplantation requires certain prerequisites. Authors have suggested PDL cells, root maturity, recipient site, patient age, and surgeons' skills as important prerequisites for successful autotransplantation $[12,18,19]$. In particular, an intact PDL, a long root trunk of the donor tooth, and age $<40$ years are more important for the management of teeth with alveolar bone resorption. An intact PDL attached to the donor tooth plays an important role in the formation of alveolar bone [9]. The vitality of PDL cells can be maintained by atraumatic harvest of the donor tooth, handling of the tooth from the crown portion to avoid scraping the root surface, and minimization of extra-alveolar time. The root shape of the donor tooth is also an important factor. The furcation of teeth with multiple roots and a short root trunk can be easily exposed after transplantation into bone with advanced periodontal disease, and such teeth tend to develop periodontal pockets in that region. Teeth with a single root or coneshaped multiple roots and a long trunk are more suitable for autotransplantation to replace teeth with advanced periodontal disease. Sugai et al. [20] indicated that younger patients aged $<40$ years showed a significantly better prognosis compared with older patients; therefore, patient age is important for a good prognosis. Age is related to the restoration of vitality and regeneration of paratransplantal tissue $[21,22]$ and a higher level of periodontal tissue regeneration is required when the tooth is transplanted in an extraction socket in a region with advanced periodontal disease.

According to Clokie et al. [23] the recipient site should fulfill the following prerequisites: adequate alveolar bone support in all dimensions, adequate attached keratinized tissue, and absence of inflammation. However, in the present case, the recipient site had inadequate alveolar bone and tooth-attached keratinized tissue because of severe periodontitis. Furthermore, the inflammation could not be completely eliminated before surgery because the tooth was mobile and the inflammation site showed furcation involvement. The positive results obtained in this case seem to suggest that autotransplantation may be successful even in patients without ideal recipient site conditions, provided that other factors related to the donor tooth and patient are fulfilled. This case report shows the possibility of using autotransplantation to replace missing teeth in regions with severe alveolar bone resorption. In this case, the transplanted tooth could be maintained without complications for 4 years, with a dramatic increase in the level of surrounding alveolar bone, particularly on the buccal and distal sides. Therefore, autotransplantation can be an optimal treatment option for the replacement of teeth with advanced periodontal disease if the conditions of the donor tooth and patient are appropriate and the vitality of the PDL attached to the donor tooth is maintained. To evaluate the effectiveness of autotransplantation in regions with alveolar bone resorption, further studies with control and experimental groups may be necessary.

\section{Conclusion}

This case report shows the possibility of using autotransplantation to replace missing teeth in regions with severe alveolar bone resorption. In this case, the transplanted tooth could be maintained without complications for 4 years, with a dramatic increase in the level of surrounding alveolar bone, particularly on the buccal and distal sides. Therefore, autotransplantation can be an optimal treatment option for the replacement of teeth with advanced periodontal disease if the conditions of the donor tooth and patient are appropriate and the vitality of the PDL attached to the donor tooth is maintained. To evaluate the effectiveness of autotransplantation in regions with alveolar bone resorption, further studies with control and experimental groups may be necessary.

\section{Acknowledgement}

The authors declare no competing interests or sources of funding. All authors have approved the submission of this manuscript and have agreed to ensure the integrity of the work.

\section{References}

1. Tanaka T, Deguchi T, Kageyama T, Kanomi R, Inoue M, et al. (2008) Autotransplantation of 28 premolar donor teeth in 24 orthodontic patients. Angle Orthod 78: 12-19.

2. Apfel H (1950) Autoplasty of enucleated prefunctional third molars. J Oral Surg (Chic) 8: 289-296.

3. Miller HM (1951) Tooth transplantation; report of case. J Oral Surg (Chic) 9: 68-69.

4. Yoshino K, Kariya N, Namura D, Noji I, Mitsuhashi K, et al. (2012) Risk factors affecting third molar autotransplantation in males: a retrospective survey in dental clinics. J Oral Rehabil 39: 821-829.

5. Andreasen JO, Paulsen HU, Yu Z, Bayer T, Schwartz O (1990) A longterm study of 370 autotransplanted premolars. Part II. Tooth survival and pulp healing subsequent to transplantation. Eur J Orthod 12: 14-24.

6. Reich PP (2008) Autogenous transplantation of maxillary and mandibular molars. J Oral Maxillofac Surg 66: 2314-2317.

7. Mejare B, Wannfors K, Jansson L (2004) A prospective study on transplantation of third molars with complete root formation. Oral Surg Oral Med Oral Pathol Oral Radiol Endod 97: 231-238.

8. Chagas e Silva MH, Lacerda MF, Chaves M, Campos CN (2013) Autotransplantation of a mandibular third molar: a case report with 5 years of follow-up. Braz Dent J 24: 289-294.

9. Park JH, Tai K, Hayashi D (2010) Tooth autotransplantation as a treatment option: a review. J Clin Pediatr Dent 35:129-135.

10. Tsukiboshi M (2002) Autotransplantation of teeth: requirements for predictable success. Dent Traumatol 18:157-180.

11. Thomas S, Turner SR, Sandy JR (1998) Autotransplantation of teeth: is there a role? Br J Orthod 25: 275-282.

12. Andreasen JO (1981) Periodontal healing after replantation and autotransplantation of incisors in monkeys. Int J Oral Surg 10:54-61. 
Citation: Shim JS, Park JH, Shin JH (2017) Autotransplantation for the Management of Teeth with Severe Alveolar Bone Resorption: A Case Report. Dentistry 7: 441. doi:10.4172/2161-1122.1000441

Page 4 of 4

13. Chiapasco M, Casentini P, Zaniboni M (2009) Bone augmentation procedures in implant dentistry. Int J Oral Maxillofac Implants 24: 237-259.

14. Jensen SS, Terheyden $\mathrm{H}$ (2009). Bone augmentation procedures in localized defects in the alveolar ridge: clinical results with different bone grafts and bone-substitute materials. Int J Oral Maxillofac Implants 24 218-236.

15. Andersson L, Andreasen JO, Day P, Heithersay G, Trope M, Diangelis AJ, et al.(2012). International Association of Dental Traumatology guidelines for the management of traumatic dental injuries: 2. Avulsion of permanent teeth. Dent Traumatol 28: 88-96.

16. Andreasen JO, Paulsen HU, Yu Z, Bayer T (1990) A long-term study of 370 autotransplanted premolars. Part IV. Root development subsequent to transplantation. Eur J Orthod 12: 38-50.

17. Nethander G (1994). Periodontal conditions of teeth autogenously transplanted by a two-stage technique. J Periodontal Res 29: 250-258.
18. Cohen AS, Shen TC, Pogrel MA (1995) Transplanting teeth successfully: autografts and allografts that work. J Am Dent Assoc 126: 481-485.

19. Nimcenko T, Omerca G, Varinauskas V, Bramanti E, Signorino F, et al. (2013) Tooth auto-transplantation as an alternative treatment option: A literature review. Dent Res J (Isfahan) 10: 1-6.

20. Sugai T, Yoshizawa M, Kobayashi T, Ono K, Takagi R, et al. (2010) Clinical study on prognostic factors for autotransplantation of teeth with complete root formation. Int J Oral Maxillofac Surg 39: 1193-1203.

21. Urbanska DK, Mumford JM (1980) Autogenous transplantation of nonroot-filled maxillary canines: a long-term follow-up. Int Endod J 13: 156-163.

22. Oksala E, Kallioniemi H (1997) A longitudinal clinical and radiographic study of autotransplantation of the maxillary canine. Proc Finn Dent Soc 73: 117-125.

23. Clokie CM, Yau DM, Chano L (2001) Autogenous tooth transplantation: an alternative to dental implant placement? J Can Dent Assoc 67: 92-96. 